\title{
Multiple Time Scales Analysis of Precipitation in Hotan, China
}

\author{
Jiqing Lv \\ Graduate student for Master Degree, Xi'an University of Technology \\ Xi'an 710048, China \\ Tel: 86-29-82312028_E-mail: lvjiqiang0721@gmail.com \\ Bing Shen \& Shuhong Mo \\ Faculty of College of Water Resources and Hydropower Engineering \\ Xi'an University of Technology \\ Xi'an 710048, China
}

\begin{abstract}
The wavelet transform and detrended fluctuation analysis (DFA) were introduced briefly. Then DFA and discrete wavelet were used to analyze the fluctuation characteristics of precipitation in Hotan from 1954 to 2003. It was shown by analysis with the methods of de-trend fluctuation and wavelet transform that the precipitation in Hotan will decreased in near future for several years, and will last 21 months at least.
\end{abstract}

Keywords: Hotan, Precipitation, DFA, Wavelet analysis

\section{Introduction}

Wavelet analysis is a subject which developed on the basis work of Y. Meyer, S. Mallat and I. Daubechies in 1986, as the epoch-making development results of Fourier analysis. P. Kumar and Foufoula-Gegious introduced orthogonal wavelet transformation to hydrology in 1993, which has made considerable achievements, mainly in the time-scale hydrological analysis, and so on. In 1993, P. Kumar and Foufoula- Gegious studied the space-scale precipitation and vibration characteristics by means of orthogonal wavelet transformation, which shows that there is a variety of components in the precipitation scale of the self-similarity and the time scale (You Wei-hong et al., 1999).

De-trend fluctuation analysis (DFA) is widespread in physics, chemistry, biology and other natural phenomena and even the climate system, in particular the considerable power measurement function which can be used to examine the complexity of the sequence of rainfall, as the basis theory and the law Practical ways in semi-arid area of rainfall research. Peng (C K Peng et al., 1993) etc. introduced the DFA approach in testing of related properties in long-chain molecules, and soon be used in many fields, such as DNA sequencing, physiology, the stock market, cloud structure, economic time series, and physics. It can also be used in climate research. DFA has a significant advantage in analysis of trends and the composition of the noise sequence scale, which depicts the relevant characteristics of the object sequence, and so on. But analysis on the de-trend fluctuation and long-range correlation to describe the characteristics of rainfall is in its infancy (Penzel et al., 2003)

Hotan oasis is located on the southern margin of the Tarim Basin in Xinjiang, the region precipitation is scarce, evaporation is dramatic, light-rich geothermal resources, belong to a typical continental arid climate(Shen Bing et al., 2003). Irrigated oasis and natural desert oasis form the green corridor which is the basic element of socio-economic development and the ecological environment development of Hotan Area. Water resources is the basic elements of survival and sustainable development for the region (Abdirahman. Halik et al., 2006). In this paper, the changes in the characteristics of precipitation from 1954 to 2003 are studied by means of wavelet and de-trend fluctuation analysis.

\section{Methods}

\subsection{Wavelet theory}

Wavelet theory outlined in the climate signal function $\mathrm{F}(t)$ of the continuous wavelet transform, said:

$$
W_{f}(s, \tau)=\int_{R} f(t) \bar{\psi}(s, \tau) d t
$$

Where, $W_{f}(s, \tau)$ is the wavelet coefficient. The wavelet can be defined as $\left|W_{\mathrm{f}}(s, \tau)\right|^{2}$ to reflects the energy size of the signal amplitude. In practical applications, time series are often discrete, the discrete form is: 


$$
W_{f}(s, \tau)=\Delta t \sum_{i=1}^{n} f(i \Delta t) \psi\left(\frac{i \Delta t-b}{a}\right)
$$

Wavelet coefficients can reflect variation characteristics of the system in the time scale corresponding to the period of rainfall. The positive wavelet coefficients correspond to the period of rainfall on the high side, the negative wavelet coefficients correspond to the period of the low rainfall (Huang Qing-qing et al., 2007). Morlet wavelet functions were selected as the generating function in this paper.

2.2 The detailed formulae description of detrended fluctuantion analysis was given as follows:

Here $X_{i}$ is the time series, $i=1,2,--; N$

$$
\bar{X}=\frac{1}{N} \sum_{i=1}^{N} X_{i}
$$

First, the series is integrated

$$
Y(i)=\sum_{i=1}^{N}\left(x_{i}-\bar{x}\right)
$$

The integrated series is separated into $N_{h}$ boxes with equal length $h$. That is $N_{h}=\operatorname{int}(N / h)$. And no overlaped segments are in the boxes.

In order to make full use of the data, we do the separated step from tail to head again. So, $2 N_{h}$ segments are obtained altogether.

Next, for each interval $v, v=N_{h}+1 \ldots 2 N_{h}$. Here $Y_{h}(i)$ is the fitting line for segment $v$.

$$
Y_{h}(i)=Y(i)-P_{v}(i)
$$

Then the de-trend fluctuation function is:

$$
F(h)=\sqrt{\frac{1}{2 N_{h}} F^{2}(v, h)}
$$

Finally,repeating the procedure described above. If time series $X_{i}$ is of long range correlation, then

$$
F(h) \sim h^{a}
$$

The index $\alpha$ is called generalized DFA exponent, which reflects the sequence of long-range correlation.

The scale index $\alpha$ meaning (Luciano Telesca et al., 2004 \& Ping Zhou et al., 2007):

a) $1>a>0.5$, if the DFA exponent is between 0.5 and 1 , the change trend will continue as the the former.

b) $0<a<0.5$, If its DFA exponent is less than 0.5 , the serie is a antipersistence sequence.

c) If the DFA exponent equals 0.5 , the series is a random sequence.

\section{Results and Conclusions}

Changing trend analysis of precipitation time series

The results show that the distribution of long year average precipitation in the Hotan has an obvious annual variation as shown in Figure 1 and Figure 2. The annual average precipitation was $35.7 \mathrm{~mm}$ (from 1954 to 2003), and changed from $3.4 \mathrm{~mm}$ (1985) to $100.9 \mathrm{~mm}$ (1987), there is a increasing trend from 1975-2003, but a slow decreasing trend before 1975.

\subsection{DFA analysis of Hotan precipitation series}

Figure 3 is the Cumulative differential curve of precipitation time series. Figure 4 is the generalized Hurst exponents $a$ using DFA Method, where $a_{1}=0.596, a_{2}=0.4081$.

The scaling index $a_{2}=0.4081$, shows that the series is a anti-persistence sequence in large-scale. And the precipitation will reduce in large-scale; $a_{l}=0.596$, which shows that the decreasing trend will continue at least 21 months.

\subsection{Wavelet analysis of Hotan precipitation time series}

The curves of wavelet variance(Figure 5) shows that primary cycles of annual precipitation in past 50 years are separately 27, 13 and 4 years. The wavelet transformation coefficient (Figure 6) shows that 13 years cycle mainly existed before the 1990s and then reduced. The 27 years and 4 years period existed between the mid-1950s and the early 21 st century. These express that the time series of annual precipitation in Hotan has gone through the process that small size year cycle weaken while mid-sized and large years cycle enhanced in the past 50 years. In particular the wavelet transformation coefficient of quasi-27-year period is developing.

The positive wavelet coefficients correspond to the period of rainfall will increase and the negative wavelet coefficients 
correspond to the period of low rainfall. Hotan in recent years, a 27-year period of negative wavelet coefficients is developing, which indicates the future may be less precipitation in the region.

\subsection{Conclusion}

It was shown by analysis with the methods of de-trend fluctuation and wavelet transform that the precipitation at Hotan will decrease in near future for several years, the trend will be lasting for, at least, 21 months.

\section{References}

Abdirahman. Halik, \&Kirimja. (2006). Study on water resources utilization and ecological environmental building of Hotan river Xin jiang. Areal research and development,25, 111-114.

Peng C K,Buldyrev SV,\&Havlin S,et al. (1994). Mosaic organization of DNA nucleotides. Phys Rev E,49,1685,1689.

Huang, Qing-qing,\&Huang,Mao-dong et al. (2007). Meizhou in the last 50 years of rainfall characteristics. Guangdong Meteorology, 29, 37-38.

Luciano Telesca,Gerardo Colangelo, \& Vincenzo Lapenna et al. (2004). Fluctuation dynamics in geoelectrical data: an investigation by using multifractal detrended fluctuation analysis. Physics Letters A, 332, 398-404.

Penzel,T.,Kantelhardt, J.W.,Grote, L.,Peter, \& J.H., Bunde. (2003). A Comparison of Detrended Fluctuation Analysis and Spectral Analysis for Heart Rate Variability in Sleep and Sleep Apnea. IEEE Trans Biomed Eng, 50, 43-1151.

Zhou, Ping, Li, Feng, Liu, Wen-Yan and Yang, Miao. (2007). Fractal analysis in normal EEG and epileptic EEG of rats. IFMBE Proceedings, 1266-1269.

Shen, Bing, Huang, Ling-mei et al. (2003). Study on the water cycling characteristics of Hotan oasis during the second half of last century. Hydraolic Engineering, 5, 78-83.

You, Wei-hong, Qi, Ming-hui, Duan, Xu. (1999). Wavalet tarnsforms' applications to researches on model of short range. Plateau Meteorology, 18, 39-46.

Van der Geer,J., Hanraads, J. A. J.,\&Lupton R. A. (2000). The art of writing a scientific article. Journal of Scientific Communications, 163, 51-59.

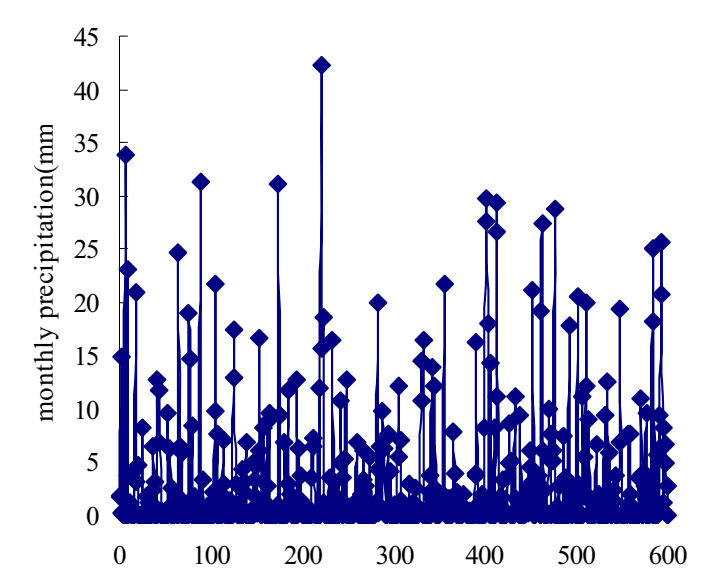

Figure 1. Monthly series process of precipitation in Hotan

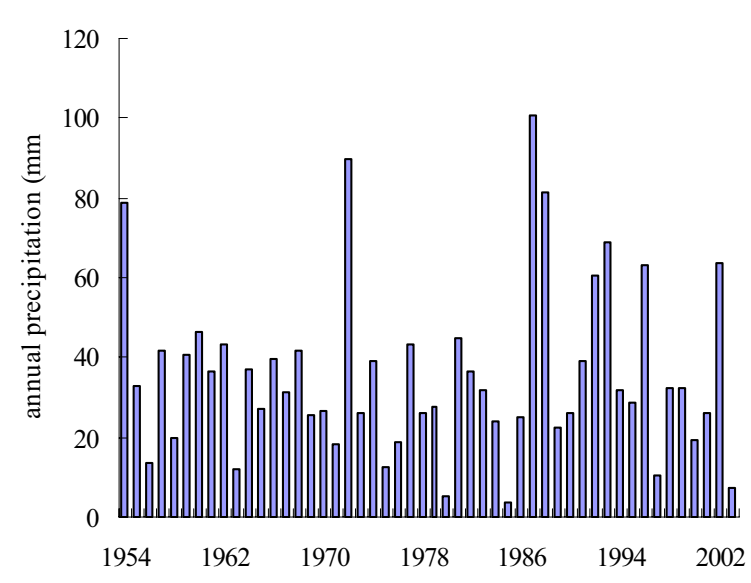

Figure 2. Yearly series process of precipitation in

Hotan 


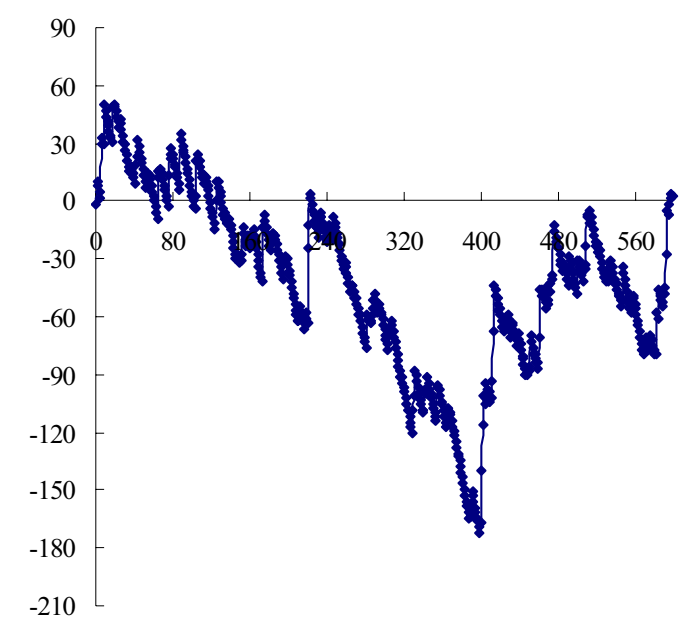

Figure 3. Cumulative differential curve of precipitation series

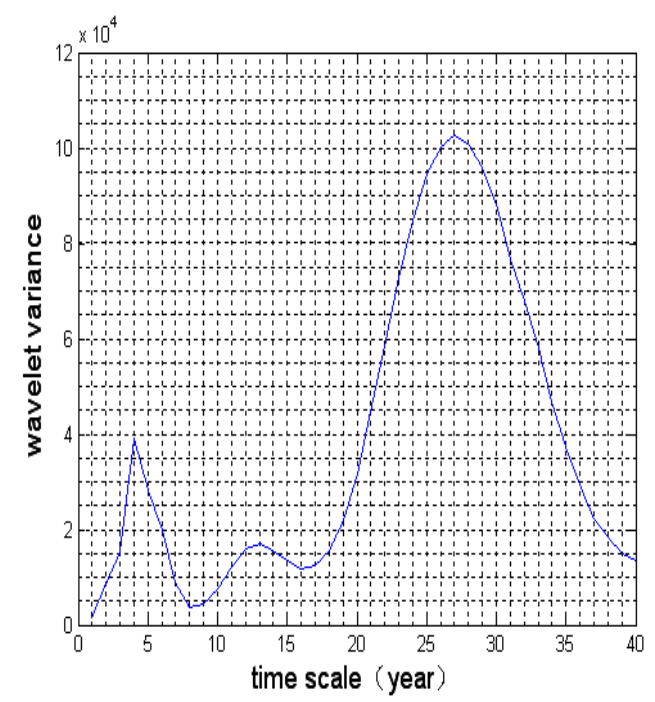

Figure 5. wavelet variance diagram

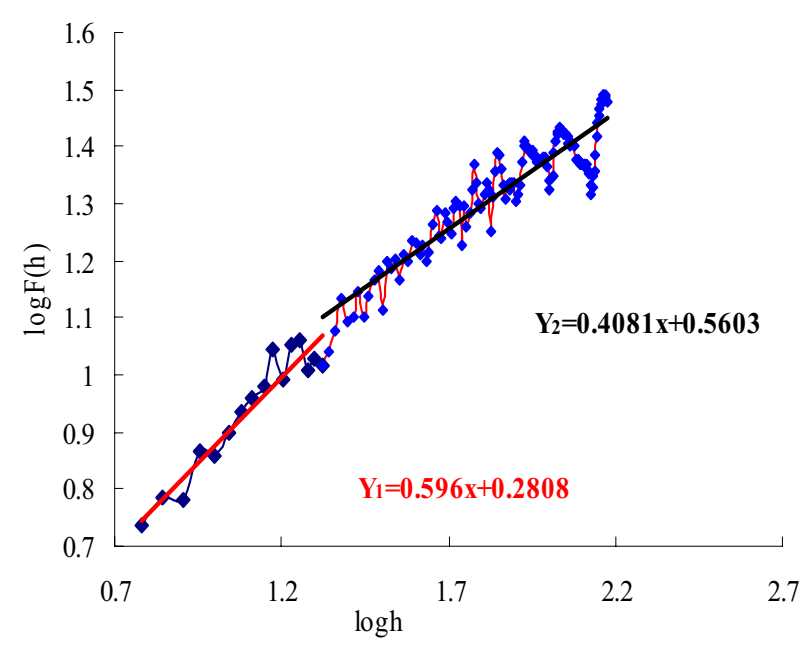

Figure 4. The detrended fluctuation of the precipitation series

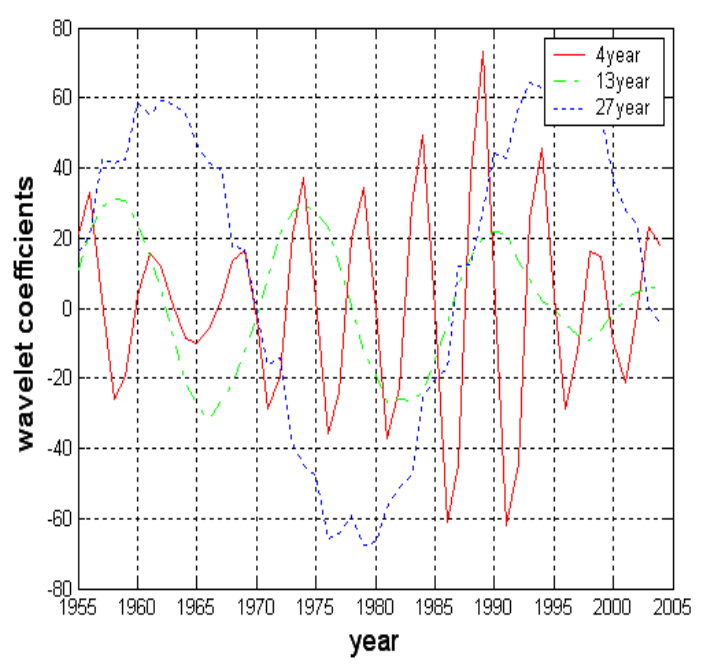

Figure 6. wavelet transformation coefficient diagram 